\title{
Use of free-form surface to simulate local refractive index changes in plastic lenses measured using polariscopic device
}

\section{Uso de superficies free-form para simular los cambios locales de indice de refracción en lentes de plástico a partir de su medida polariscópica}

\author{
J. Arasa15, C. Pizarro ${ }^{1,2}$, P. Blanco ${ }^{1}$ \\ 1 :Center for Sensors Instruments and Systems Development (CD6), Universitat Politècnica de \\ Catalunya, Rambla de Sant Nebridi 10, E08222 Terrassa, Spain \\ 2: SnellOptics ${ }^{T M}$, Carrer Sant Quirze 91,5e2a. E08221 Terrassa, Spain \\ E-mail: josep.arasa@upc.edu $\quad$ S: Sedóptica Member
}

Received: 02/12/2016 Accepted: 10/07/2017

DOI: 10.7149 /OPA.50.3.49060

\begin{abstract}
:
In this paper we propose a method to convert the local variations of refractive index into local changes of one surface of the lens, which then can then be described as a free-form surface, easy to introduce in conventional simulation tools. The proposed method tries to provide an equivalent model of the lens with refractive index variations where the index changes have been replaced for local thickness variations on the lens' surface. The local thickness variations can be fitted at a freeform description that holds all the local index variations through the OPD (Optical patch difference) using a B-Spline description and a simplex algorithm to find the best fitted surface.
\end{abstract}

Key words: free-form surfaces, index variations, polarization, plastic lenses.

\section{RESUMEN:}

En este trabajo se propone un método para convertir las variaciones locales de índice de refracción en cambios locales de una superficie de la lente con el fin de poder evaluar si su calidad es suficiente para el objetivo con que fue diseñada. La nueva superficie de la lente puede describirse como una superficie free-form, fácil de introducir en herramientas de simulación convencionales. El método propuesto trata de proporcionar un modelo equivalente de la lente para simular las variaciones del índice de refracción como cambios locales de espesor de la lente a través de la OPD (diferencia de camino óptico) inducida por el cambio local del índice de refracción, el ajuste del mapa de OPD se realiza mediante una descripción B-spline de la nueva superficie y utilizando un algoritmo Simplex para hallar la mejor descripción

Palabras clave: superficies free-form, variaciones de índice, polarización, lentes de plástico.

\section{REFERENCES AND LINKS / REFERENCIAS Y ENLACES}

[1] Thorlabs, generic optical supplier, http://www.thorlabs.de/newgrouppage9.cfm?objectgroup_id=16

[2] G-S plastic Optics, plastic optic supplier, http://gsoptics.com/product/index.php

[3] K. Matsusaka; S. Ozawa; R. Yoshida; T. Yuasa; Y. Souma "Ultracompact optical zoom lens for mobile phone", Proc. SPIE 6502, Digital Photography III, 650203 (2007); https://doi.org/10.1117/12.702712

[4] Ledil, plastic optic supplier, http://www.ledil.fi/

[5] Neotronic, illumination supplier, http://www.neotronic.com/EN/kathod-lenses-for-power-leds.html

[6] Carclo, plastic optic supplier, http://www.carclo-optics.com/

[7] P. Obreja, P. Cristea, A. Dinescu, R. Rebigan, "Micro and nanoscale patterning of polymers for microoptics", Proceedings of the European Optical Society Annual Meeting 2010 (EOSAM 2010), ISBN 9783-00-030509-2. Parc Floral de Paris, France, (2010) 
[8] D. Cristea, P. Obreja, A. Dinescu, G. Konstantinidis, R. Rebigan, C. Kusko, "Fabrication of 3D micro and nanostructures for photonic applications", Proceedings of 4M Conference- eds. B.Fillon, C.KhanMalek, S.Dimov, Research Publishing, p.263-266, ISBN 978-981-08-6555-9 (2010) https://doi.org/10.3850/978-981-08-6555-9.168

[9] M. Kusko, C. Kusko, D. Cristea, "Analytical solution for the distribution of scattering elements in edgelit backlight units", 2nd EOS Conference on Manufacturing of Optical Components (EOSMOC 2011) at the World of Photonics Congress, Munich, Germany, (2011)

[10] P.T. Tang, R. Sobiecki, M. Hansen, C. Ravn, C. Baum, "Replication of large scale embossed optical polymer surfaces”, ICOMM proceedings, pp. 431-435. Tokyo. (2011)

[11] S. Hiroyuki" Interferometric measurement of the refractive-index distribution in plastic lenses by use of computed tomography" Appl. Opt. 41, 5317-5325 (2002). https://doi.org/10.1364/A0.41.005317

[12] Y.L. Chen, H-C Hsieh, W.T Wu,W.Y. Chang, and D.C. Su, "Alternative method for measuring the fullfield refractive index of a gradient-index lens with normal incidence heterodyne interferometry" Appl. Opt. 49 6888-6892 (2010) https://doi.org/10.1364/A0.49.006888

[13] J. Arasa, D. Mayershofer, J. Romero "In-line polariscopic checking of plastic molded-injected lenses: preliminary results" Optical Measurement Systems for Industrial Inspection IX, Spie Procedings Volume Number: 9525 ISBN: 9781628416855, Munich (2015)

[14] N. Domínguez; D. Mayershofer; C. Garcia; J. Arasa, "Accurate polarimeter with multi-capture fitting for plastic lens evaluation," Opt. Eng. 55, 024102 (2016). https://doi.org/10.1117/1.0E.55.2.024102

[15] G. Farin, “Curves and Surfaces for CAGD” 5th edition, Morgan Kaufmann, San Francisco 2002

[16] P. Jester, C. Menke and K. Urban. "B-Spline Representation of Optical Surfaces and its Accuracy in a Ray Trace Algorithm” Appl. Opt. 50, 822-28 (2011) https://doi.org/10.1364/A0.50.000822

[17] B. Narasimhan, P. Benitez, Juan C. Miñano, Julio Chaves, D. Grabovickic, Milena Nikolic, J. Infante, "Design of freeform aplanatic systems" Proc. SPIE. 9629, Optical Systems Design 2015: Illumination Optics IV, 96290C. (2015)

https://doi.org/10.1117/12.2190882

[18] G. Farin and D. Hansford “The Essentials of CAGD” AK Peters, Ltd. Publishers, ISBN: 1-56881-123-3 (2000)

[19] LightPath Technologies, 2603 Challenger Tech Court, Suite 100, Orlando, USA, http://www.lightpath.com/

[20] Zemax, LLC, 10230 NE Points Dr. Suite 540, Kirkland, WA 98033 USA, http://www.zemax.com/

[21] C. de Boor. “A practical guide to Splines.” Ed. Springer Verlag. ISBN 0-387-95366-3 New York (2001).

[22] J. A. Nelder, R. Mead. "A simplex method for function minimization.” The computer journal Vol. 7 pp. 308-313. (1965)

[23] Y. S. Kim. "Refined Simplex method for data fitting." Astronomical Data Analysis Software and Systems VI, A.S.P. Conference Series, Vol. 125, 1997, Gareth Hunt and H. E. Payne, eds., p. 206.

\section{Introduction}

In recent years thermoplastic injected lenses have reached the required standard quality to be part of high performance optical systems. Injected lenses are no longer a lower quality product and they are now included into quality systems and services [1][2] ranging from automotive applications to the latest generation of optical zooms for mobile telephones [3]. A plastic lens can be manufactured by three different processes. First, it can be cut and mechanically polished from a block of material, as done in conventional glass lenses. In this case, the block of material has not undergone any volume strains, as in the glass case, so the lenses have equivalent optical quality to its glass equivalents. The second possible process is in situ polymerization (typically in e.g. Allyl-diglycol-carbonate (CR39) and equivalent thermostable materials). This technique is fully in use for ophthalmic lenses; where the monomer is injected at low pressure and the polymer undergoes a polymerization reaction to obtain the final lens. The process is typically slow; usually they need around 2 hours in the best case, and the properties of the lens 
differ from glass lenses as the characteristics of glass ones because of the density variations inside the lens caused by the polymerization process. Thus, the polymerization time is set to be smooth, typically lasting several hours, so the variations may be done almost negligible, making these lenses a relevant alternative to glass lenses. Finally, the third process is injection molding, used in thermoplastic lenses [4][5][6]. Due to the fast solidification process involved, these lenses present the greatest local differences in refractive index in their volume, making them the most relevant candidates for in-line polariscopic quality control.

The constituent polymers with thermoplastic properties applied to optics are Poly-methyl methacrylate (PMMA), Polycarbonate (PC), Cyclic-olefin-copolymer (COC), and Styrene acrylonitrile (SAN). All of them move from solid to liquid state at temperatures between $200^{\circ} \mathrm{C}$ and $300^{\circ} \mathrm{C}$, which are slow enough to make them useful for production purposes. Once in the liquid state they can be injected into a mold, adopting the shape of the cavity, and refrigerated to get back to solid state. The cost of this process is very small, and the material remainder and rejected lenses may be recycled. However, their large advantages regarding their fast and cheap production turn into disadvantages when inaccurate processing turns them into low-quality lenses.

The origin of the problem is the same manufacturing process. The solidification of the thermoplastic induces a fast change form a liquid to a solid state, so a thermodynamic change of state is induced. As the density of liquid and solid thermoplastic are different, and the time in the transition is very short (usually less than 5 seconds) a dynamic, small scale process in the solidification appears, inducing stress, birefringence and local changes of density and refractive index.

This dynamic transition starts in the surface in contact with the mold skin and ends inside the core of the lens. Thus, the solidification process of the injected thermoplastic lenses (whether PMMA or PC) involves a volumetric change which affects the refractive index, turning the lens into a gradient index (GRIN) with a particular index profile, which cannot be neglected if quality imaging applications are targeted.

The two main disadvantages of plastic lenses against glass ones are the change of its optical properties with temperature at near-ambient temperatures, and its dependence on geometrical shape (being thickness the most relevant factor) [7][8][9][10]. There are also some additional dependences to be considered in the parameters linked to the injection process, where internal stress and volume reduction may play a very important role in the final optical performance.

Even in plastic lenses using optimized injection procedures, refractive index presents smooth variations from the internal to the external regions, which appear when the thermoplastic shrinks during the solidification process by introducing variations in shape and surface. This effect is well known since the very beginning of the use of thermoplastics, and has so far been considered as a manufacturing defect. Studies on the GRIN profiles induced in injected lenses using the generic injection method [11], and some others propose methods for accurately measuring the induced refractive index variations [12] [13] [14].

However, once a good method to estimate the internal refractive index changes is available, a new problem appears when they need to be included in an optical simulation software tool. In this paper we propose a method to include the internal refractive index variations in the lens in simulation tools by transforming them in the equivalent optical path changes, and then using this computed optical path difference (OPD) to calculate a free-form surface [15], [16], [17] which enables to compensate for the refractive index changes in the lens.

\section{Method}

The proposed method intends to provide an equivalent model of the lens to simulate the refractive index variations in the lens. To do so, we will use a lens with homogeneous refractive index and the nominal geometric parameters to those of the plastic "GRIN" lens, but shaping one of its surfaces with a free-form curve, so the local change in the lens thickness introduces an optical path difference equivalent to the refractive index variation in the same local coordinates.

The method, see Figure 1, starts with a proposed lens (with the nominal surface's shapes). This lens including the free-form surface will be named from now on the "final lens". At its initial stage, it has the same geometrical parameters of the initial lens; the plastic "GRIN" one, but the material of the lens shows a homogenous refractive index. In the next step the wavefront leaving the initial and the end lenses are calculated. Such wavefront measurement needs be carefully done in the same plane, as it is in this plane where they will be compared in the next step. 
The local OPD difference between the wavefronts at that plane may then be calculated, and used to obtain the local sagitta of the surface assuming a homogeneous refractive index, which will be added/subtracted to the final lens. When this OPD is added/subtracted to the final lens the same wavefront expected from the initial lens in the selected wavefront plane will be obtained. To use the second surface of the final lens as the plane where the OPD is computed is the best option, as in these conditions the OPD can be directly translated to thickness changes, by just dividing the optical path changes by the value of the refractive index.

The last step of the method is a fitting process to generate a new continuous surface for the end lens, which can be carried out using a B-spline curves description [18] to fit such a surface, as usually the refractive index variations which appear have no observable symmetry.

$\underline{\text { Initial lens (with index variations) }}$

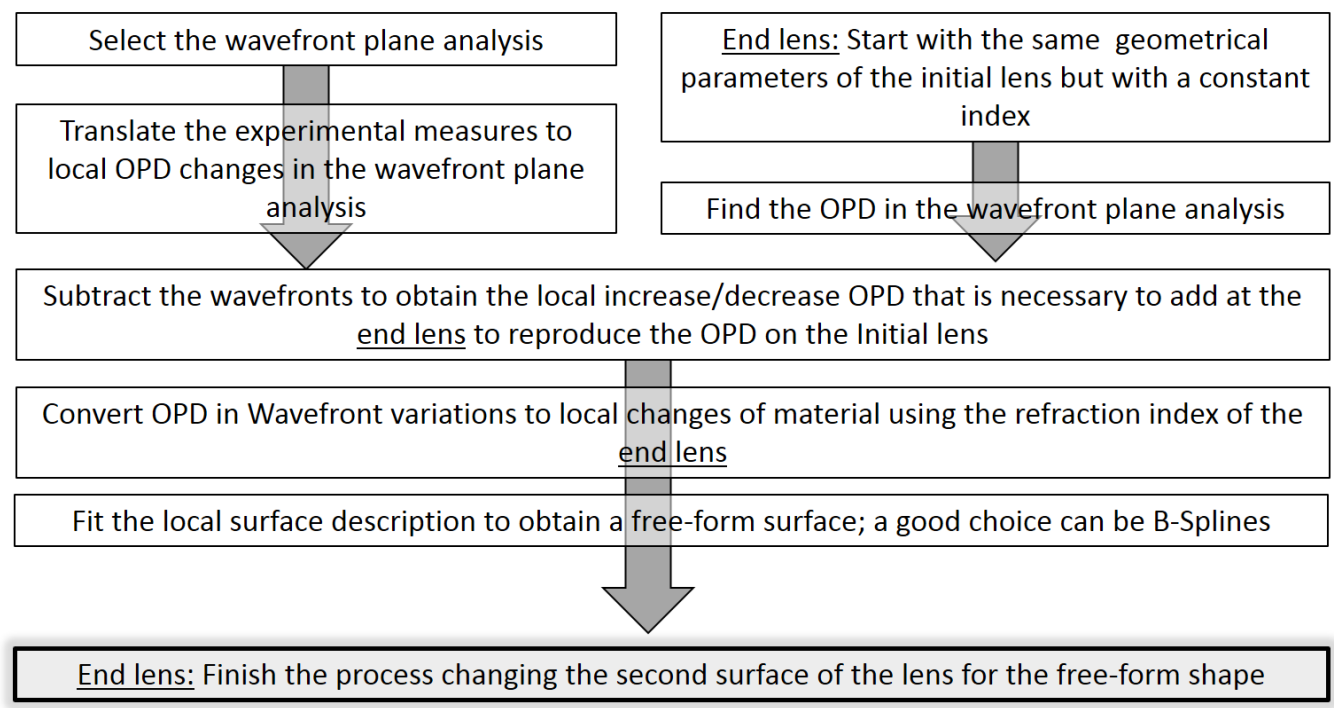

Figure 1: Flux diagram of the method to replace the second surface of a lens with homogenous refractive index with a free-form surface, so the wavefront obtained is equivalent to one obtained in the original lens with a gradient distribution

\section{Checking of the method}

Prior to apply the proposed method in a real lens the proposed method has been tested using a commercial GRIN lens with known index profile. Using this strategy, the experimental measures of the refractive index was substituted by the analytical expression of the index profile. The lens was selected from the commercial catalogue of LightPath ${ }^{\mathrm{TM}}$ [19] technologies with reference GPX-10-30. The analytical expression of its profile is described as

$$
n=\sum_{i=0}^{11} n_{i}\left(\frac{z+\Delta z}{z_{\max }}\right)^{i}
$$

Being $\mathrm{n}$ the refractive index value along $\mathrm{Z}$ axis; $\mathrm{z}$ the coordinate value along the lens axis where $\mathrm{n}$ will be computed, and $\Delta \mathrm{z}$ the step using during the simulation. For this lens $z_{\max }=9,4 \mathrm{~mm}$ and the $n_{i}$ numerical values are presented in Table 1. The main optical features of the lens are presented in Figure 2. The aperture stop of the lens is placed on the second surface. Under this conditions the OPD for an object at infinity is evaluated using Zemax ${ }^{\mathrm{TM}}$ [20] (Figure 3.a) obtaining a maximum OPD value of 0,035 $\lambda$ (@546 $\mathrm{nm}$ ) in the exit pupil, which has a diameter of $10 \mathrm{~mm}$. 


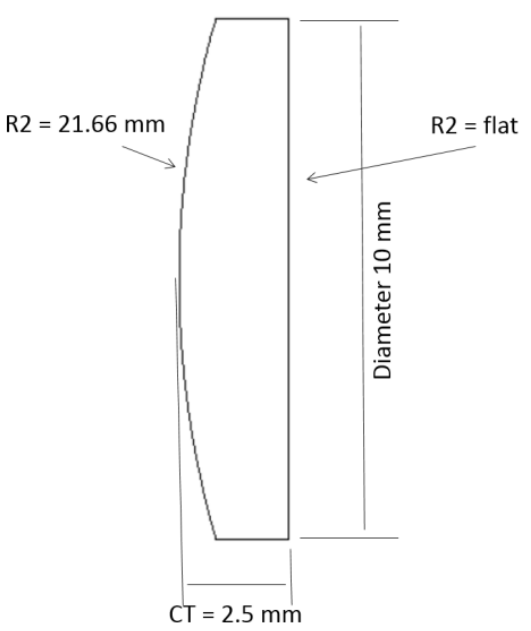

Glass description

G23SFN

Diameter

$10 \mathrm{~mm}$

Radius first surface(R1)

$21.66173 \mathrm{~mm}$

Radius second surface(R2)

Infinity (Flat)

Central thickness (CT)

$2.5 \mathrm{~mm}$

Effective focal length (EFL)

$30 \mathrm{~mm}$

Figure 2.- Scheme and main optical parameters of GPX-10-30 from LightPath ${ }^{\mathrm{TM}}$

Table 1.- Coefficients of equation (1)used to obtain the refractive index profile of GPX-10-30 lens

\begin{tabular}{|c|l|c|l|}
\hline$i$ & \multicolumn{1}{|c|}{$n_{i}$} & $i$ & \multicolumn{1}{|c|}{$n_{i}$} \\
\hline 0 & $1.7758298 \mathrm{E}+000$ & 6 & $9.2133053 \mathrm{E}+001$ \\
\hline 1 & $-6.3476076 \mathrm{E}-002$ & 7 & $-5.3438915 \mathrm{E}+001$ \\
\hline 2 & $6.8687048 \mathrm{E}-001$ & 8 & $-2.4823518 \mathrm{E}+001$ \\
\hline 3 & $-6.7605889 \mathrm{E}+000$ & 9 & $6.8676506 \mathrm{E}+001$ \\
\hline 4 & $2.8904287 \mathrm{E}+001$ & 10 & $-4.8879704 \mathrm{E}+001$ \\
\hline 5 & $-6.9313554 \mathrm{E}+001$ & 11 & $1.2759259 \mathrm{E}+001$ \\
\hline
\end{tabular}

As final lens we will calculate another lens with the same geometrical parameters of the initial lens (Figure 2 ), but changing the glass description to a constant refractive index of 1,7601. The OPD of this new lens is presented in Figure $3 \mathrm{~b}$ and has been chosen as the standard lens for our purposes. The OPD images are enough to show the homogenous refractive index lens does not have the same optical behavior of the initial lens. Such differences may be easily appreciated from the tangential cuts of OPD in Figures 4a and $4 \mathrm{~b}$
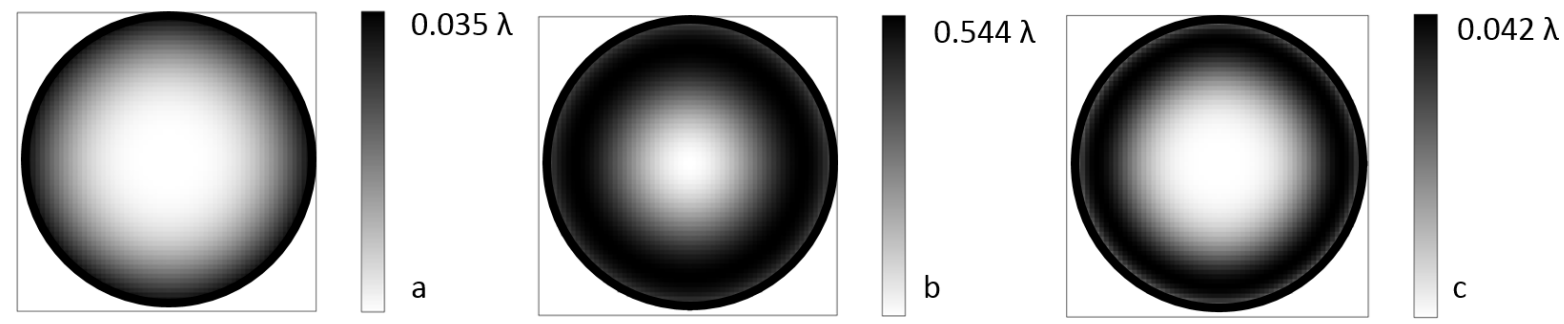

Figure 3, OPD at the exit pupil (second surface of the lens) at $\lambda=546 \mathrm{~nm}$, grey scale of OPD in $\lambda$, box dimensions $10 \times 10 \mathrm{~mm}$, a) Initial GRIN lens GPX-10-30 b) Final lens when the GRIN profile is changed by a constant index of $1.760101 \mathrm{c}$ ) Final lens with constant index and a second free-form surface added. 


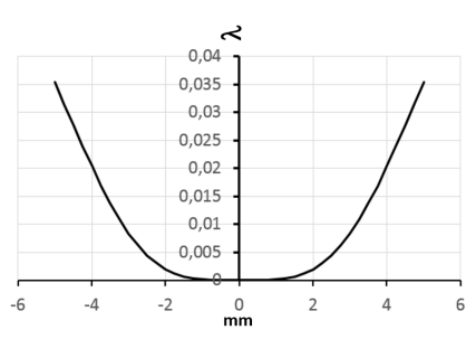

a

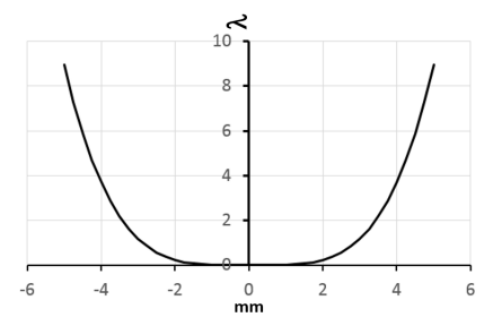

b

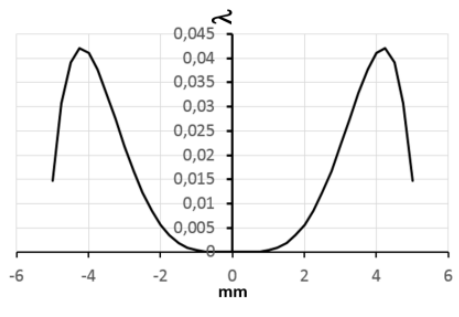

C

Figure 4, Sagittal cuts of the OPD in the exit pupil, placed on the second surface of the lens at $\lambda=546 \mathrm{~nm}$, vertical scale in $\lambda$, horizontal scale in mm, a) Initial lens GPX-10-30 b) Final lens with GRIN profile replaced by a constant index of $1.760101 \mathrm{c}$ ) Final lens with the free form shape added at the second surface.

The next step is to subtract the sagittal cuts of the OPDs obtained from the initial and final lenses and use the difference to create a free-form shape which yields a final wavefront equivalent to that of the initial lens.

The final lens has the same geometrical parameters of the initial lens presented in Figure 2, but the GRIN profile has been replaced by a constant refractive index lens with an ad hoc local thickness distribution (see Figure 5) to yield an equivalent wavefront shape A fitting procedure of local thickness was performed to obtain an analytical form from the simulated values obtained with Zemax ${ }^{\mathrm{TM}}$. The target function was

$$
z=a r^{4}+b r^{2} \quad \text { where } r^{2}=x^{2}+y^{2}
$$

Where $\mathrm{z}$ is now the height of the OPD surface taking as origin the apex of the OPD surface, and $\mathrm{x}$ and $\mathrm{y}$ are the coordinates of the plane normal to the optical axis. Using a least squares method [21] strategy to find the coefficients of Equation 2 we obtain a value of $a=1,0179^{*} 10^{-5} \mathrm{~mm}^{-3}$ and a value of $b=1,8470^{*} 10^{-6} \mathrm{~mm}^{-}$ 1 , with a correlation coefficient $\left(r^{2}\right)$ of 1,000 , meaning the equation passes on all data values. As data is not experimental, error bars or further statistical analysis is not required.

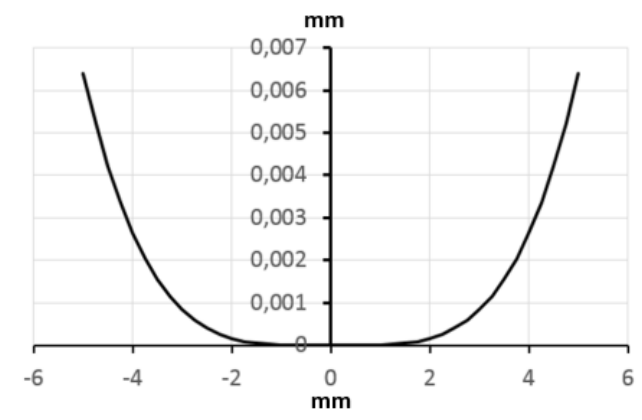

Figure 5: Local thickness distribution in the second surface of the final lens to obtain an equivalent optical behavior. The graph reproduces a sagittal cut $(\mathrm{y}=0)$, with $\mathrm{X}$ axis in the horizontal and $\mathrm{Z}$ axis in vertical. Scales are in $\mathrm{mm}$.

Now we can compute again the OPD in the same plane (see Figures 3c and 4c). Note that the obtained OPDs are not exactly equal; at the border of the OPD function, in the $4-5 \mathrm{~mm}$ distance from the center, OPD values decrease, where they were expected to stay at top values. Deviations, however, deviate around $0,005 \lambda$ of the initial lens. Such differences will be seen to be not relevant for our proposal, see for instance the spot diagrams of Figure 7.

In order to set and extra accuracy test of the method, the MTF sagittal plots of initial and final lenses until a $400 \mathrm{l} / \mathrm{mm}$ frequency for an object placed on axis and at infinity were calculated (Figure 6). Table 3 completes the comparison of the initial and final lenses with a table presenting the RMS values of spot size for different object positions. It is relevant to notice how both the values on axis and at full field are of comparable magnitude in both lenses. 


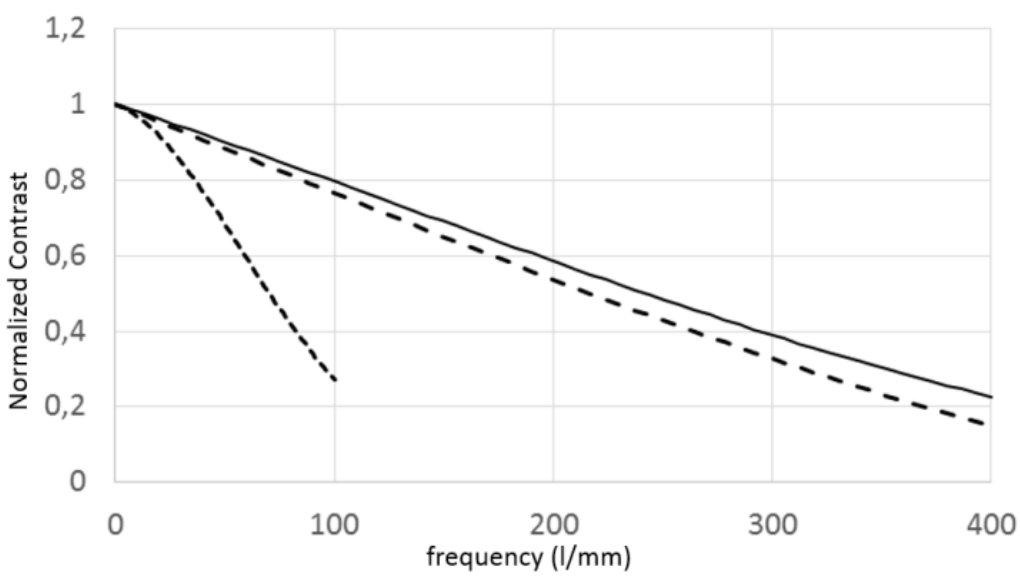

Figure 6, Sagittal MTF representation up to a frequency of $400 \mathrm{l} / \mathrm{mm}$ for the initial lens (solid line) and final lenses (long dash line). In the graph also shows the MTF values of the final lens without the inclusion of a free-form surface (short dash).

Table 2: RMS values of the spot diagrams for the initial and end lens. To do this comparison the image plane was always refocus to obtain the minimum RSM value for the axis position of the object.

\begin{tabular}{|r|l|l|r|r|}
\hline \multirow{2}{*}{ Id } & \multirow{2}{*}{ Object placed at } & \multirow{2}{*}{ Field value } & \multicolumn{2}{|c|}{ RMS spot radius (microns) } \\
\cline { 4 - 5 } & & Initial lens & \multicolumn{1}{c|}{ Final lens } \\
\hline 1 & Infinity & Axis & 0,025 & 0,465 \\
\hline 2 & Infinity & 5 degrees & 35,745 & 44,351 \\
\hline 3 & 5 times EFL & Axis & 2,754 & 2,718 \\
\hline 4 & 5 times EFL & $10 \mathrm{~mm}$ high & 29,379 & 34,939 \\
\hline 5 & 2 times EFL & Axis & 22,055 & 28,488 \\
\hline 6 & 2 times EFL & 5 mm high & 111,659 & 132,540 \\
\hline
\end{tabular}

The set of spot diagrams corresponding to the objects described in Table 2 are presented in Figure 7 . The degree of accuracy in the reproduction of optical performance between the Initial and final lenses is presented, showing the feasibility to substitute a GRIN lens by a lens with constant index with a free-form second surface, in this case described by a $4^{\text {th }}$ degree polynomial equation.

\section{Results}

We present the results of the method on a plastic lens manufactured by injection molding, and measured using an in-house polariscope to obtain the residual local refractive index variations. The lens selected was one of the worse, but functional, lens of the injected batch. The lens, which has a meniscus shape lens was injected molded in PMMA and made in ASCAMM (a Technological Center devoted to plastic manufacture in the Barcelona area http://www.ascamm.com) in Figure 8 presents the geometrical parameters of the manufactured lens, and Figure 9 the lens itself and the tooling used, to do the injecting molding process. The measurements of the local refractive index variations were done using an in-house built polarimeter based on a transmissivity polariscopic arrangement shown in Figure 10. 


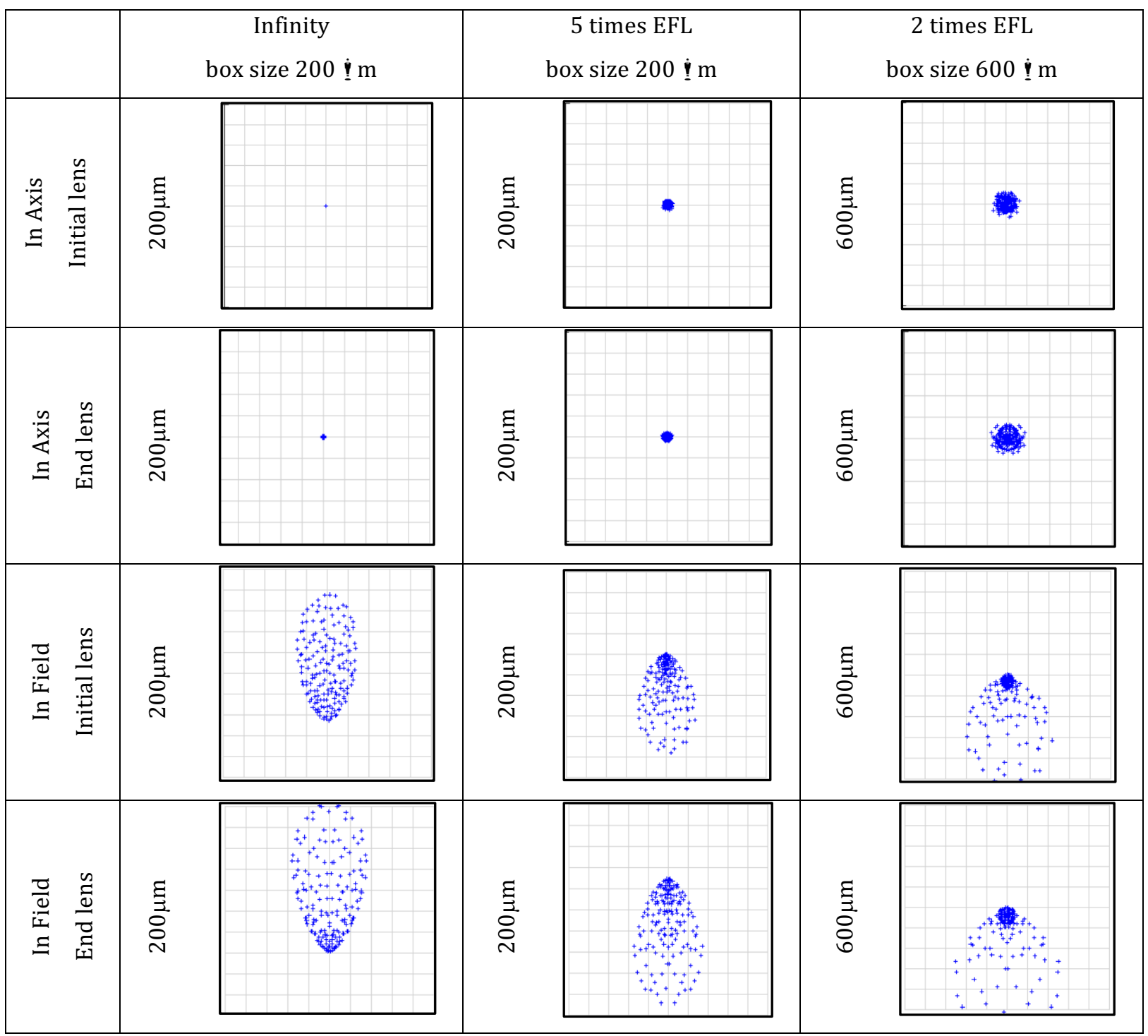

Figure 7. Spots diagrams for the initial and final lenses. Box size is $200 \mu \mathrm{m}$ for the infinity and 5 times EFL cases, and $600 \mu \mathrm{m}$ for 2 times EFL. In all the cases the size and structure of the spots are similar showing an equivalent optical behavior.

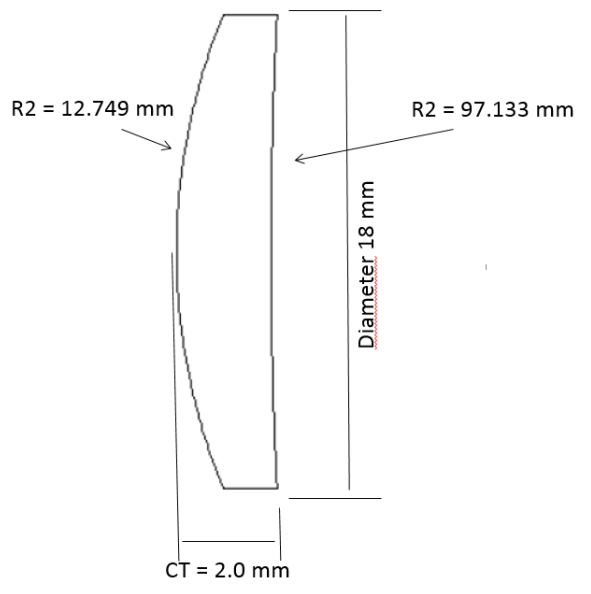

Glass description

$\begin{array}{ll} & \text { PMMA } \\ \text { Diameter } & 18 \mathrm{~mm}\end{array}$

Radius of $1^{\text {st }}$ surface (R1)

$12.749 \mathrm{~mm}$

Radius of $2^{\text {nd }}$ surface (R2)

$97.133 \mathrm{~mm}$

Central thickness (CT)

$2.0 \mathrm{~mm}$

Effective focal length (EFL)

$29.6 \mathrm{~mm}$

Figure 8.- Optical specifications of the injected molded lens 


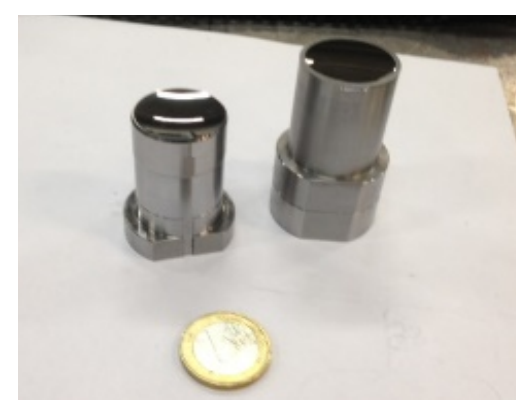

a

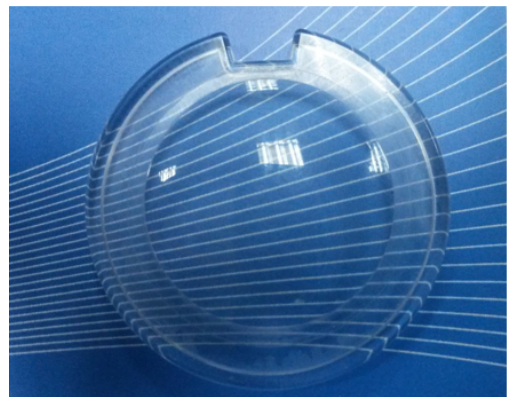

b

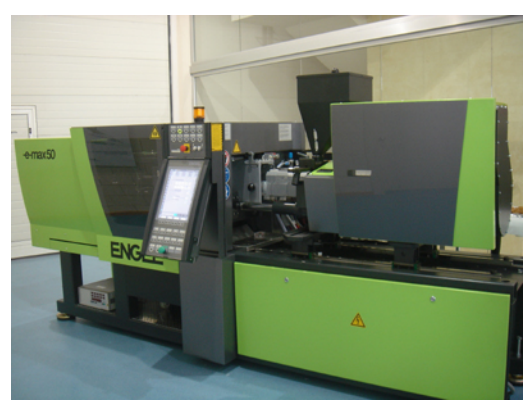

$\mathrm{C}$

Figure 9.- a) Optical parts of the mold used to inject the sample lens; b) Obtained lens; the ring around the central part is for mechanical support purposes $\mathrm{c}$ ) injecting molding machine used to make the lens

The local refractive index variations were measured using an in-house polarimeter; this polarimeter, see Figure 10, uses a collimated light beam to illuminate the sample lens, which is dipped in a liquid with matching refractive index. The light crossing the sample is collected with an afocal system and its image recorded on a CMOS image sensor. The selection of an afocal system enables to capture the image keeping the same lateral magnification when small changes in the sample position are introduced. The experimental device, shown in Figure 10, comprises a vertical stage and several perpendicular plates where different elements are assembled. The entire device is controlled by a laptop computer which can manage the LEDs for illumination, the stage motors and the CMOS camera.

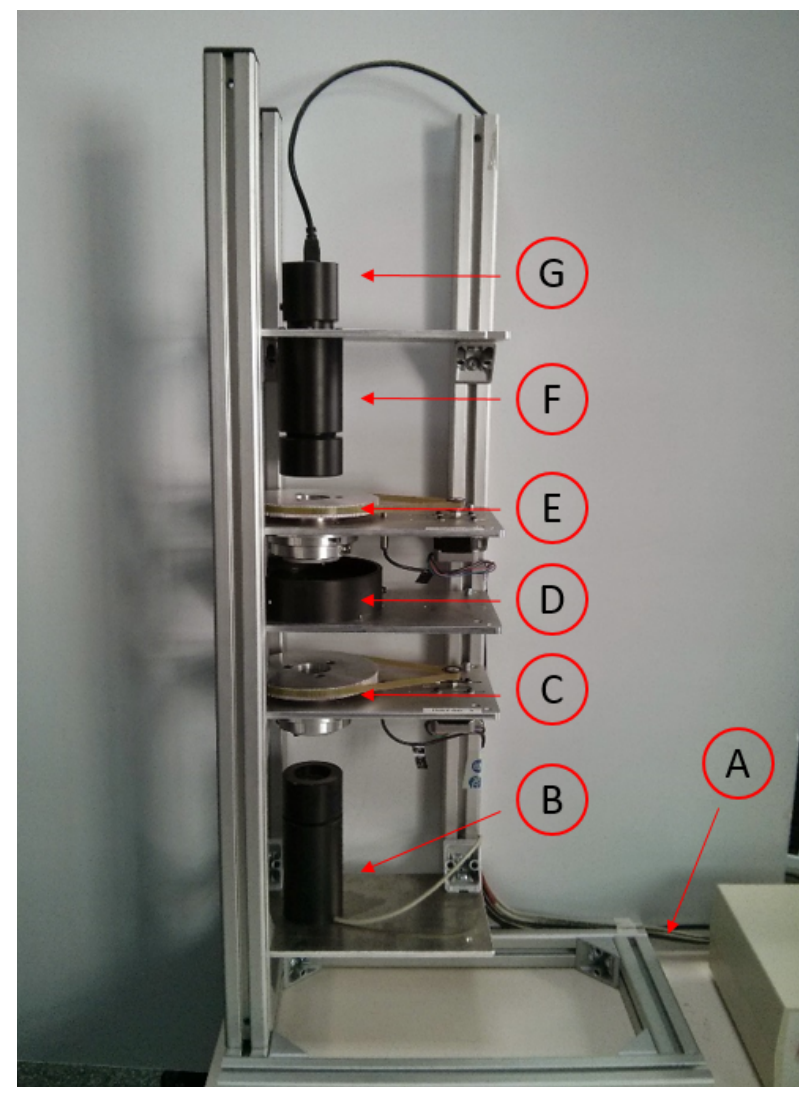

(a)

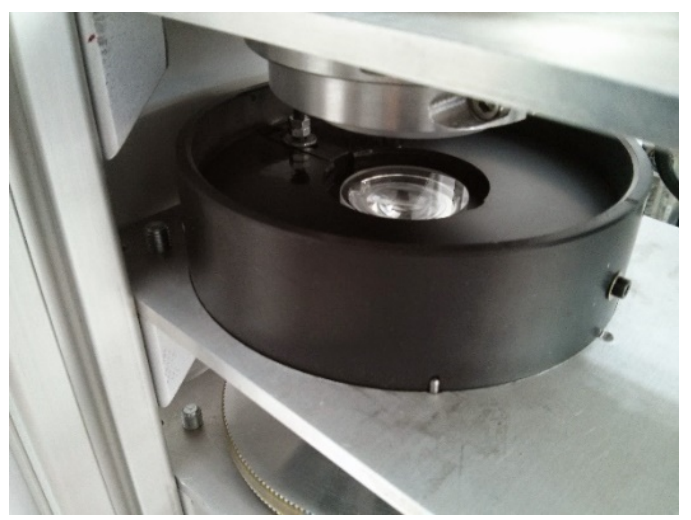

(b)

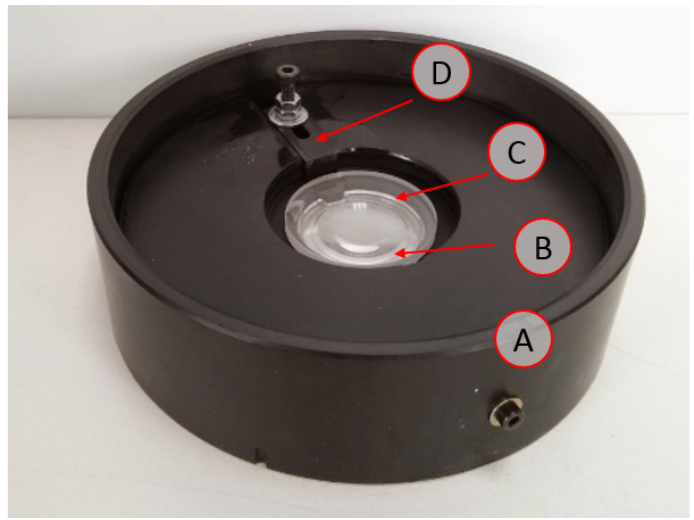

(c)

Figure 10; Picture of the experimental setup; a) Polarimetric bench, bottom to top (A) Wires to connect with controller and laptop computer; (B) Light source, the illumination device uses a LED sources @626 nm (red)) that can be electronically switched and a $100 \mathrm{~mm}$ effective focal lens to collimate the light; (C) Polarizer rotation stage, the orientation of the polarizer is also controlled from the laptop; (D) Cuvette, more in detail in picture 2b and 2c; (E) Rotation stage for the analyzer, as the orientation of the analyzer is also controlled from the laptop, (F) Afocal imaging system to ensure a constant magnification under small errors in positioning; and

(G) CMOS sensor; b) Detail of the cuvette in the polariscopic bench c) Structure of the cuvette; (A) holder, the bottom part is on borosilicate, (B) lens under test placed inside the cuvette, the cuvette here is empty, otherwise it is not possible to see it (C) cover of the cuvette - flat window of N-BK7 glass (D) fixation of the cover; b) and c), detailed view of the setup. 
The original optical behavior of the lens measured with the setup in figure 10 can be seen in Figure 11, where a 2D map of the OPD is shown (Figure 11a) and a sagittal cut of the OPD is also presented (Figure $11 \mathrm{~b})$

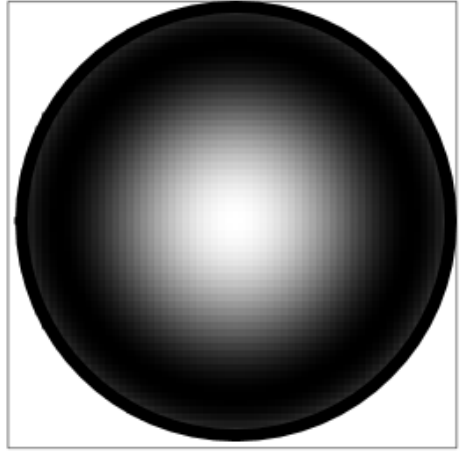

a

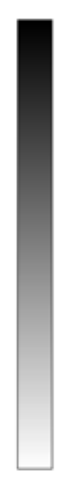

$4.03 \lambda$

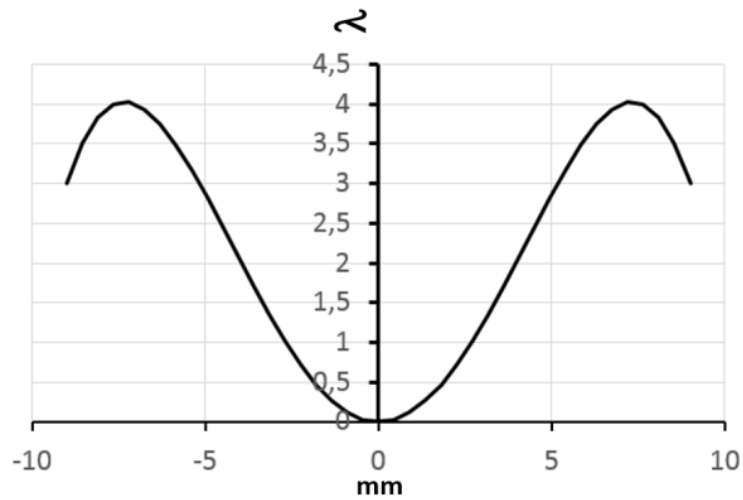

b

Figure 11. OPD of the manufactured lens for an object placed at infinity and with a wavelength of $626 \mathrm{~nm}$ a) $2 \mathrm{D}$ map, box dimensions $18 \times 18 \mathrm{~mm}$ b) Sagittal cut, vertical scale in wavelength units and horizontal scale in mm

In these conditions we can also apply the method proposed before. We start from the measurements of the polarimeter which provide us with a local refractive index variation map of the lens (Figure 12). The local changes are in $2.4 \mathrm{e}-10$ units, which are can be directly translated into an OPD map by multiplying the difference in refractive indexes by the local thickness of the lens (Figure 13a).

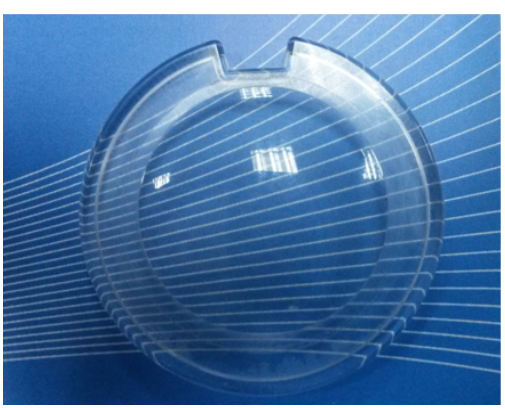

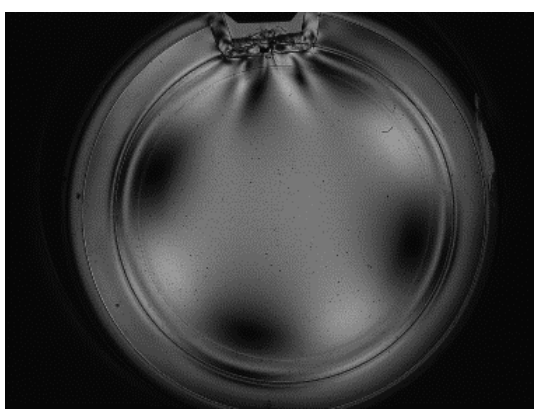

b

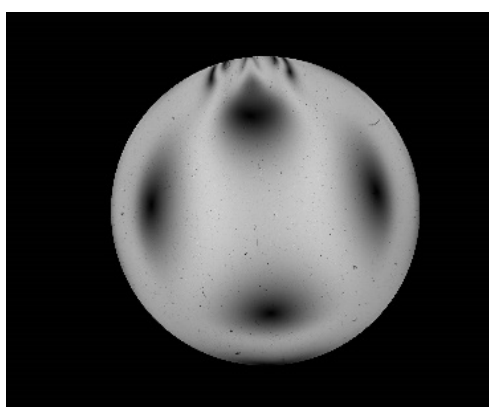

C

Figure 12.- a) Injection-molded lens provided by ASCAMM. The optically active area has a diameter of only $18 \mathrm{~mm}$, with the surrounding area used to fix the lens in the holder, b) One of the measurements obtained using the polariscope, c) Local refractive index differences; the complete image bit depth (black to white) covers $2.4 \mathrm{e}-10$ units of refractive index

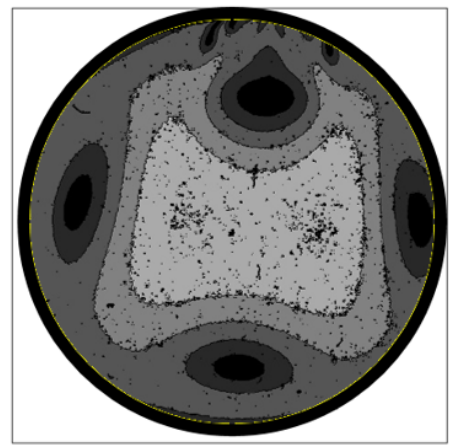

a
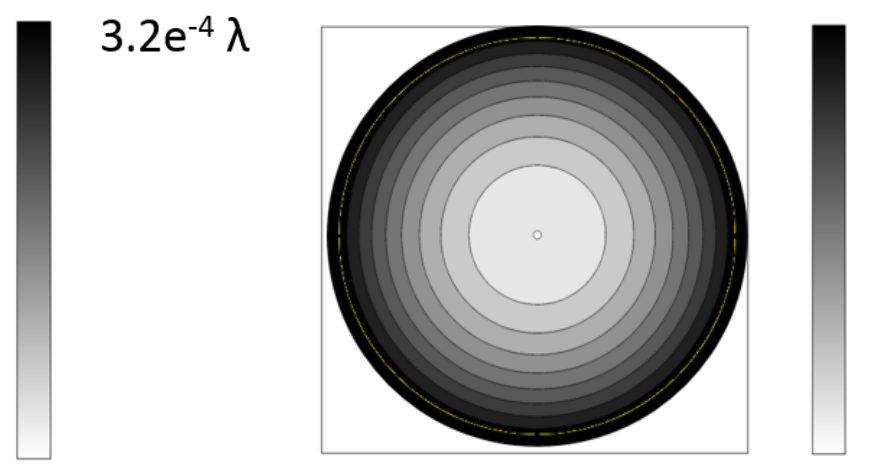

$420 \mu \mathrm{m}$

$\mathrm{b}$

Figure 13 a) Local changes of OPD measured, b) New free form surface generated using the measured local variations of OPD; size of the box $18 \times 18 \mathrm{~mm}$ 
Out of the local variations of the second surface of the lens a free-form surface was computed to compensate them and fitted to an analytical surface, in our case a uniform and symmetrical B-Spline description was used [21]. The free-form surface was obtained on a regular grid of 10x10 control points which covers all the optical area (Figure 14).

As the free-form surface of the final lens must be obtained by fitting, a merit function and an optimization strategy need be defined. In our example the merit function was set so the absolute difference between the original values and the values of the fitted surfaces was less than 0,01 microns in all the points used for the fitting.

$$
M F=\operatorname{abs}(\operatorname{dist}((\text { raw data })-(\text { surface data })))<0,01
$$

for $\forall$ data values

In order to shorten the computation time in the fitting of Equation 3 only a quarter of the 586.000 useful experimental points collected in the optical area were used.

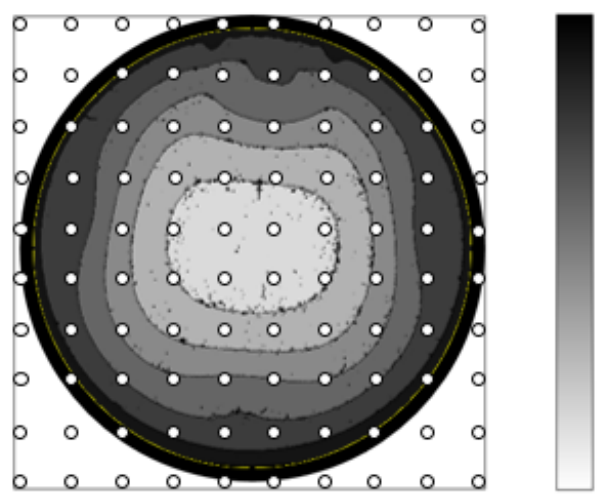

\section{$420 \mu \mathrm{m}$}

Figure 14.- Control points for B-Spline adjustment. The figure presents the points superimposed to the 2D representation of the second surface of the lens; size of the box $18 \times 18 \mathrm{~mm}$

Table 3.- $\mathrm{Z}$ value (in microns) of the control points of the B-Spline used to fit the second surface to the measured local thickness variations. A uniform and symmetrical B-Spline description was used. The $\mathrm{X}$ and $\mathrm{Y}$ values of the control points were determined for the selected grid sample, which in this case had values goes from $-9 \mathrm{~mm}$ to $9 \mathrm{~mm}$ in 10 equally spaced steps. The reference of the B-

Spline control points is taken on a plane perpendicular to the Z-axis and tangent to the apex of the second surface of the lens

\begin{tabular}{|c|c|c|c|c|c|c|c|c|c|c|}
\hline & 1 & 2 & 3 & 4 & 5 & 6 & 7 & 8 & 9 & 10 \\
\hline 1 & 0 & 0 & 0 & 0 & 423,02 & 266,76 & 0 & 0 & 0 & 0 \\
\hline 2 & 0 & 0 & 381,67 & 299,02 & 257,72 & 258,25 & 300,61 & 384,31 & 0 & 0 \\
\hline 3 & 0 & 381,67 & 257,72 & 175,18 & 133,93 & 134,46 & 176,76 & 260,36 & 385,37 & 0 \\
\hline 4 & 0 & 299,02 & 175,18 & 92,70 & 51,49 & 52,02 & 94,29 & 177,82 & 302,72 & 0 \\
\hline 5 & 423,02 & 257,72 & 133,93 & 51,49 & 10,30 & 10,83 & 53,08 & 136,57 & 261,42 & 427,77 \\
\hline 6 & 266,76 & 258,25 & 134,46 & 52,02 & 10,83 & 11,35 & 53,61 & 137,10 & 261,95 & 428,30 \\
\hline 7 & 0 & 300,61 & 176,76 & 94,29 & 53,08 & 53,61 & 95,88 & 179,40 & 304,31 & 0 \\
\hline 8 & 0 & 384,31 & 260,36 & 177,82 & 136,57 & 137,10 & 179,40 & 263,00 & 388,01 & 0 \\
\hline 9 & 0 & 0 & 385,37 & 302,72 & 261,42 & 261,95 & 304,31 & 388,01 & 0 & 0 \\
\hline 10 & 0 & 0 & 0 & 0 & 427,77 & 428,30 & 0 & 0 & 0 & 0 \\
\hline
\end{tabular}

As important as the merit function selected it is the optimization strategy used to find the best positions of the control points, in our case we have used a Simplex Algorithm strategy [22] [23]. Table 3 presents the $\mathrm{z}$-coordinates of the control points used to describe the surface using B-Spline description. Coordinates $\mathrm{x}$ 
and y are linked to the cell position, where each cell correspond to an incremental step of 1,8 mm, which contains a variable number of experimental points. Table 4 presents the residual errors of the fitting process, with the values shown in each cell of the table corresponding to local differences between the average value of the fitted surface (described using B-Splines) and the average of value of the sample points in the same cell. Such residual values are presented on the lens diameter in Figure 15, showing fitting errors below $0.01 \mu \mathrm{m}$ in all cases.

Table 4: Residual values (in microns) at the selected control point between the measured data and the nominal surface shape. All values are positive due to the merit function used in the fit. In all the values are below 0,01 microns

$\begin{array}{ccccccccccccc} & \mathbf{1} & \mathbf{2} & \mathbf{3} & \mathbf{4} & \mathbf{5} & \mathbf{6} & \mathbf{7} & \mathbf{8} & \mathbf{9} & \mathbf{1 0} \\ \mathbf{1} & 0 & 0 & 0 & 0 & 0,00998 & 0,00989 & 0 & 0 & 0 \\ \mathbf{2} & 0 & 0 & 0,00584 & 0,00672 & 0,00720 & 0,00716 & 0,00665 & 0,00576 & 0 \\ \mathbf{3} & 0 & 0,00587 & 0,00719 & 0,00797 & 0,00857 & 0,00849 & 0,00813 & 0,00733 & 0,00585 & 0 \\ \mathbf{4} & 0 & 0,00672 & 0,00802 & 0,00892 & 0,00961 & 0,00954 & 0,00910 & 0,00798 & 0,00674 & 0 \\ \mathbf{5} & 0,00993 & 0,00716 & 0,00854 & 0,00952 & 0,01004 & 0,01008 & 0,00957 & 0,00867 & 0,00731 & 0,00983 \\ \mathbf{6} & 0,00981 & 0,00709 & 0,00849 & 0,00962 & 0,01003 & 0,01009 & 0,00953 & 0,00860 & 0,00717 & 0,00984 \\ \mathbf{7} & 0 & 0,00670 & 0,00817 & 0,00918 & 0,00960 & 0,00961 & 0,00903 & 0,00811 & 0,00680 \\ \mathbf{8} & 0 & 0,00571 & 0,00730 & 0,00808 & 0,00872 & 0,00865 & 0,00804 & 0,00725 & 0,00580 \\ \mathbf{9} & 0 & 0 & 0,00584 & 0,00688 & 0,00708 & 0,00725 & 0,00672 & 0,00588 & 0 \\ \mathbf{1 0} & 0 & 0 & 0 & 0 & 0,00986 & 0,00982 & 0 & 0 & 0\end{array}$
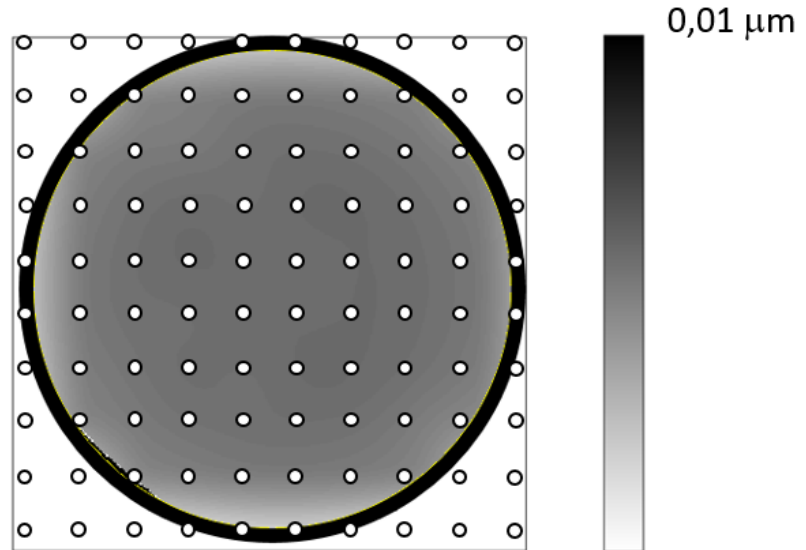

Figure 15. Residuals of the fitting in the selected control points. Numerical values can be found in Table 3. Points (in white) are superimposed to the surface.

Thus, the injected molded lens, which presented uncontrolled changes of refractive index due to the manufacturing process used, can now be exchanged by this lens of equivalent performance, with the second surface replaced by a free-form shape easy to implement in further optical simulations.

In this particular example the values of the induced changes in the refractive index where below the minimum amount relevant to noticeably impact the shape of the second surface. To validate the proposed method, we artificially raised two orders of magnitude the weight of the internal changes in refractive, so the effect in the final free-form surface was noticeable. It is stressed that such change in scale does not affect the validity of the proposed method. 


\section{Conclusions}

We have proposed a method to model in conventional optical simulation software tools the effects on optical performance of the internal refractive index changes in an injection molded plastic lens. The final lens has a constant refractive index and the same geometrical parameters of the initial lens, but its second surface is replaced by a free-form surface. In the paper we present a method to calculate such free-form surface, and we have applied it to a commercial GRIN lens and to a real PPMA injected molded lens whose internal refractive index variations have been measured using an in-house built polarimeter.

\section{Acknowledgments}

This work has been financed by the "Spanish Ministerio de Economía y Competitividad" grant DPI201238647-C02-01. 